\title{
A New Solid Deuterium Source of Ultra-Cold Neutrons
}

\author{
A. Saunders, T. Bowles, R. Hill, G. Hogan, S. Lamoreaux, L. Marek, \\ C. L. Morris, \\ S. Seestrom, W. Teasdale \\ Los Alamos National Laboratory \\ D. A. Smith, C.-Y. Liu, S. Hoedl, A. Young \\ Princeton University \\ B. Filippone, T. M. Ito, J. Yuan \\ California Institute of Technology \\ P. Geltenbort \\ Institute Laue-Langevin
}

In polarized neutron decay, the angular correlation between the neutron spin and the direction of emission of the electron is characterized by the coefficient A. Measuring A involves determining the forward-backward asymmetry of the decay beta with respect to the direction of the neutron polarization. The value of $A$, when combined with measurements of the neutron lifetime, determines the values of the vector and axial vector weak coupling constants, $G_{V}$ and $G_{A}$. The value of $G_{V}$ can also be determined by measurements of superallowed nuclear beta decay and by requiring that the Cabibo-Kobayashi-Maskawi (CKM) mixing matrix be unitary along with the measured value of other elements of the CKM matrix.

Four measurements of the A coefficient have been made recently using cold neutron beams at reactors. Each of the measurements quote an uncertainty of about $1 \%$ in the value of $A^{1,2,3,4}$. As seen in Fig. 1, the recent measurements show poor agreement both with each other and with the values of $\mathrm{G}_{\mathrm{V}}$ determined by the superallowed beta decay experiments and the requirement of CKM unitarity ${ }^{5}$. In order to resolve these discrepancies, we have proposed a new measurement of the $A$ coefficient, using a different technique from the preceding experiments both in order to have an independent set of systematic errors and to improve the precision of the measurement.

We have proposed to measure the A coefficient using Ultra-Cold Neutrons (UCN) instead of cold neutrons. UCNs, neutrons moving so slowly that they are totally externally reflected from common materials, have several properties that make them advantageous for a measurement of $\mathrm{A}$. The can be transported through bent guide tubes, so the experiment can be well shielded from any source of background. UCNs can be $100 \%$ polarized by passing them through a magnetic field gradient with a maximum field near 5 Tesla. In a system with small depolarization, essentially

CP529, Capture Gamma-Ray Spectroscopy and Related Topics: $10^{\text {h }}$ Int'l. Symp., edited by S. Wender C 2000 American Institute of Physics 1-56396-952-1/00/\$17.00 
$100 \%$ polarized UCN can be supplied to the experiment. Finally, if the UCN are produced using a spallation source instead of a reactor, they can be stored to further reduce backgrounds by only counting in the experiment between pulses of the spallation beam.

UCN can be produced by several means. The original neutrons to be converted to UCNs can be produced either at a reactor or using a spallation source. Reactors have the advantage that higher densities and rates can be achieved, while spallation sources have the advantage that they can be pulsed to allow timing information to be used as part of the experiment. In either case, the neutrons are then moderated to become cold neutrons, then further moderated to become UCNs. The final step to the Ultra-Cold state can be made either by reflecting the cold neutrons off a rotor, as at the existing sources at the Institut Laue-Langevin ${ }^{6}$ (ILL) and at LANSCE, or by using a superthermal moderator? A rotor source uses the kinematics of bouncing a particle off of a receding surface to reduce the energy of the neutrons. Our new source uses a superthermal solid deuterium moderator to convert the cold neutrons to UCN.

As described by Golub, Richardson, and Lamoreaux ${ }^{8}$, a superthermal UCN source can greatly enhance UCN production compared to other sources. In a material such as solid deuterium, cold or ultra-cold neutron interactions with the moderator are primarily by single phonon interaction. That is, a neutron can produce a phonon and lose energy (downscatter), or absorb a phonon and gain energy (upscatter). The downscattering rate depends on the density of states available for the production of phonons; the upscattering rate depends on the density of available phonons to be absorbed. If the density of phonons at UCN energies is much less than the density of states at cold neutron energies, UCN production can be greatly enhanced relative to UCN removal by upscattering. Very preliminary results at the Gatchina reactor have shown a gain in UCN densities achieved of a factor of ten using a superthermal solid deuterium source as compared to a liquid deuterium source. This type of source is referred to as "superthermal" because the temperature of the moderator is much higher than the temperature of the UCNs.

A schematic of our new source is shown in Fig. 2. The neutrons are produced by hitting a tungsten target with the $800 \mathrm{MeV}$ proton beam at LANSCE. The neutrons are trapped and multiplied in the beryllium box, and moderated by the beryllium and polyethylene held at $77 \mathrm{~K}$ to become cold neutrons. The cold neutrons are further moderated by a layer of polyethylene at $5 \mathrm{~K}$, then enter the $5-7 \mathrm{~K}$ solid deuterium in the guide at the center of the source, where they can be converted into UCN. The UCN then travel up the guide system, until they exit the source and can be trapped in a bottle or detected. A typical pulse of protons hitting the tungsten spallation target consists of about $10^{12}$ protons, and produces about $10^{3} \mathrm{UCN}$ exiting the source.

We have spent the last year exploring the interaction of the neutrons with the solid deuterium moderator, in order to learn how to design an optimized source of UCN. 
The thickness of the solid deuterium, for example, is limited by several factors. UCNs can only travel a finite distance in solid deuterium before elastically scattering. Similarly, they have only a finite lifetime before being removed by being absorbed on a deuterium atom or upscattered by absorbing a phonon. (The removal due to upscattering and absorption is expressed as a lifetime since the cross sections for these processes go as the inverse of the velocity of the neutron; therefore, one velocity independent lifetime applies to all ultra-cold neutrons.) Finally, the cold neutron flux is highest near the tungsten spallation target, so adding more deuterium, necessarily farther from the tungsten, does not produce as many additional UCN as might be expected. A naïve calculation of the distance a UCN can travel in solid deuterium, before being elastically scattered, absorbed, or upscattered, yields a value of about $8 \mathrm{~cm}$.

Fig. 3 shows the UCN density produced in the solid deuterium as a function of the volume of the deuterium. The surface area of the solid was $50 \mathrm{~cm}^{2}$; a volume of 100 $\mathrm{cc}$ therefore corresponds to a thickness of $2 \mathrm{~cm}$, for example. A fit to the data is shown, indicating that the effective path length in the solid deuterium was only about $1 \mathrm{~cm}$, not 8 the expected $8 \mathrm{~cm}$. A curve showing the expected behavior if the path length was $8 \mathrm{~cm}$ is also shown in the figure. Since the variation in cold neutron flux is a small effect, the conclusion was that either the elastic scattering length or the neutron lifetime caused by absorption or upscattering is much shorter than expected.

To separate out the effect of the elastic scattering length and the lifetime, we measured the neutron lifetime in the deuterium directly. To do this, we constructed a $\mathrm{UCN}$ bottle with a bottle lifetime significantly greater than the lifetime of the neutrons in the deuterium. The lifetime in the guide system was limited by the UCN detector on the end; the detector effectively formed a surface black to UCN on the end of the guide. We put a valve on the end of the guide with an aperture only one tenth the size of the detector face, or $5 \mathrm{~cm}^{2}$ instead of $50 \mathrm{~cm}^{2}$. Since the storage time of a bottle with an aperture is inversely proportional to the size of the aperture, adding the valve increased the storage time of the guide system (in the absence of deuterium) by a factor of ten. This meant that neutron losses from the guide system were dominated by upscattering and absorption in the deuterium, and loss to the detector was a relatively small effect. The result of these direct lifetime measurements was that the lifetime of the neutrons in the solid deuterium was much shorter than would be expected from a simple calculation of the absorption and upscattering cross sections. Of course, the elastic scattering length could also be shorter than expected, but the short lifetime dominated any such effect in the volume dependence measurements and we did not measure the elastic scattering length directly.

The lifetime of the neutrons in the deuterium could be shorter than expected for two reasons. Either the absorption or the upscattering was larger than expected. The absorption could be larger than expected because of contamination of the deuterium with other elements or with hydrogen. To eliminate the hydrogen, we used 
deuterium gas which was isotopically pure to a level of $99.9 \%$; that is, the hydrogen contamination was less than $0.1 \%$. At this level, the absorption of neutrons in the hydrogen is expected to be a small effect. To eliminate all other elements, we flowed the deuterium gas through a palladium membrane before introducing it to the guide system. Since only hydrogen and its isotopes flow through palladium, and other elements do not (to the parts per billion level), this procedure ensured that we had only deuterium and the small hydrogen contamination in the guide. Therefore, we believed the absorption of the UCNs in the deuterium was not significantly higher than predicted.

The upscattering, however, could easily be higher than predicted for two reasons. First, the upscattering rate is highly dependent on the temperature of the solid deuterium. There was considerable heat flux down the guide, since it ran in a straight line from a region of $5 \mathrm{~K}$ to a region of $300 \mathrm{~K}$. The solid deuterium was cooled from the sides and bottom by liquid helium on the outside of the stainless steel guide. If the deuterium was not thermally well coupled to the steel, it could be at a substantially higher temperature than the liquid helium cryostat. Measuring the temperature of the solid deuterium and ensuring that it is well coupled to the cold guide is an area of ongoing effort in the source development effort.

The second reason the upscattering could be greater than expected is a previously unexplored source of upscattering ${ }^{10}$. Like hydrogen, molecular deuterium has two possible spin states, called ortho and para deuterium. In hydrogen, the para state corresponds to the case where the spins of the two hydrogen nuclei are oppositely aligned; the ortho state to the case where they are similarly aligned. The para state is the ground state in hydrogen. Since the deuterium nucleus has spin 1 instead of spin , the situation is slightly more complicated. The ortho state in deuterium corresponds to total spin equal to 0 or 2 , while the para case corresponds to total spin equal to 1 . Unlike in hydrogen, in deuterium the ortho state is the ground state. The difference in energy between the two states is $7 \mathrm{meV}$. Since the typical energy of a $\mathrm{UCN}$ is a few hundred neV, the conversion energy between the two spin states in deuterium is more than sufficient to upscatter a UCN and remove it.

At room temperature, the spin states are all equally populated. Since there are twice as many ortho states as para states in deuterium, room temperature deuterium is $2 / 3$ ortho and $1 / 3$ para. Since the conversion between the spin states normally occurs very slowly (on the order of a few percent per month), the solid deuterium in the cryostat was also at the room temperature ratio. The contamination of $1 / 3$ para deuterium supplied many sites for upscattering a neutron by its converting the para deuterium to ortho and gaining the resulting $7 \mathrm{meV}$. By lowering the temperature of the deuterium in the presence of a catalyst, the deuterium can be converted to nearly all be in the ground (ortho) state. At a catalyst temperature of $15-20 \mathrm{~K}$, the ratio is about $95 \%$ ortho deuterium and $5 \%$ para deuterium. We have constructed a conversion cell, in which the deuterium can be catalyzed to the ground state. 
Studying the effect of different ratios of ortho and para deuterium on the neutron lifetime and UCN production are ongoing efforts of the source development.

The source as it now exists, with appropriately scaled up proton beam currents, is marginally capable of providing enough UCNs for the proposed A coefficient measurement. By understanding the dependence of the neutron lifetime and UCN production on such factors as the ortho/para ratio, the temperature of the solid, and the elastic scattering path length, we will be able to design a UCN source which will allow higher count rates in the experiment, higher UCN densities in a storage bottle, and further experiments at LANSCE using UCNs. We hope to conclude the UCN source development by mid 2000 , at which time we can move on to designing the final source and building the experiment.

References:

1. Klemt, E. et al., Z. Phys. C37 (1988) 179; Bopp, P. et al., Phys. Rev. Lett. 56 (1986) 919; Bopp, P. et al., Nucl. Inst. Meth. A267 (1988) 436; Dohner, J. et al., Nucl. Inst. Meth. A284 (1989) 123.

2. Erozolimskii, B.G. et al., Phys. Lett. B236 (1991) 33.

3. Schreckenbach, K. et al., Phys. Lett. B349 (1995) 427.

4. Abele, H. et al., Phys. Lett. B407 (1997) 212.

5. Dubbers, D., Nucl. Phys. A257 (1991) 239c.

6. Altarev, I. S., Sov. Phys.-JETP Lett. 44 (1986) 344.

7. Ageron, P. et al., Phys. Lett. 66A (1978) 469.

8. Golub, R., Richardson, D., and Lamoreaux, S. K. Ultra-Cold Neutrons. Adam Hilger, Bristol, 1991. 62ff

9. Serebrov, A. P. et al., Proc. Int. Symposium on Neutron Optics and Related Facilities, Kumatori, Japan-J. Phys. Soc. Jpn. 65, Suppl. A (1996) 259.

10. Liu, C.-Y., Young, A. R., and Lamoreaux, S. K. "UCN upscattering rates in a molecular deuterium crystal", submitted to Phys. Rev. $C$ for publication. 
Figure 1: Recent measurements of A compared to related measurements of the neutron lifetime, superallowed beta decay measurements, and CKM unitarity.

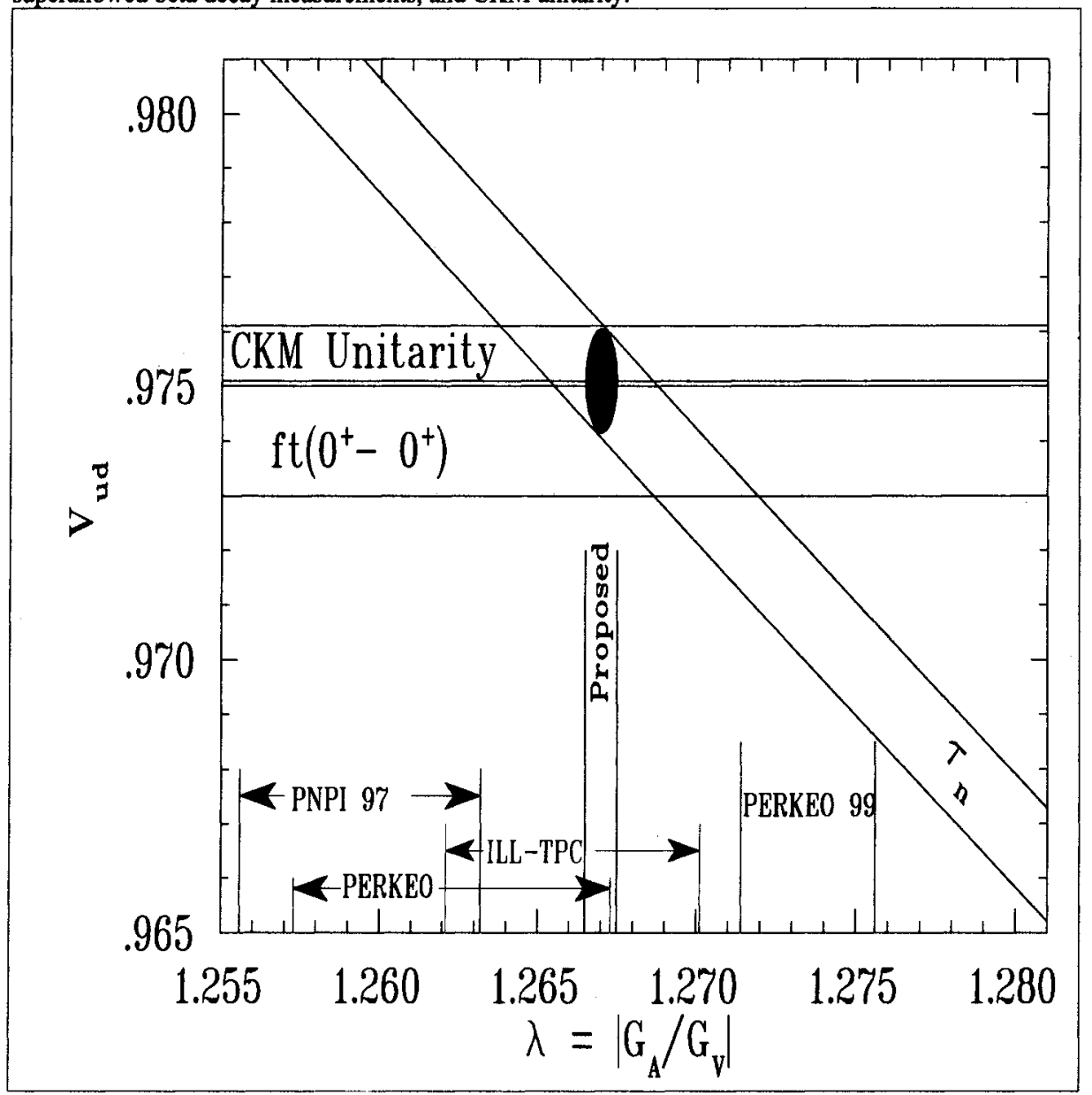


Figure 2: Schematic of new superthermal solid deuterium UCN source.

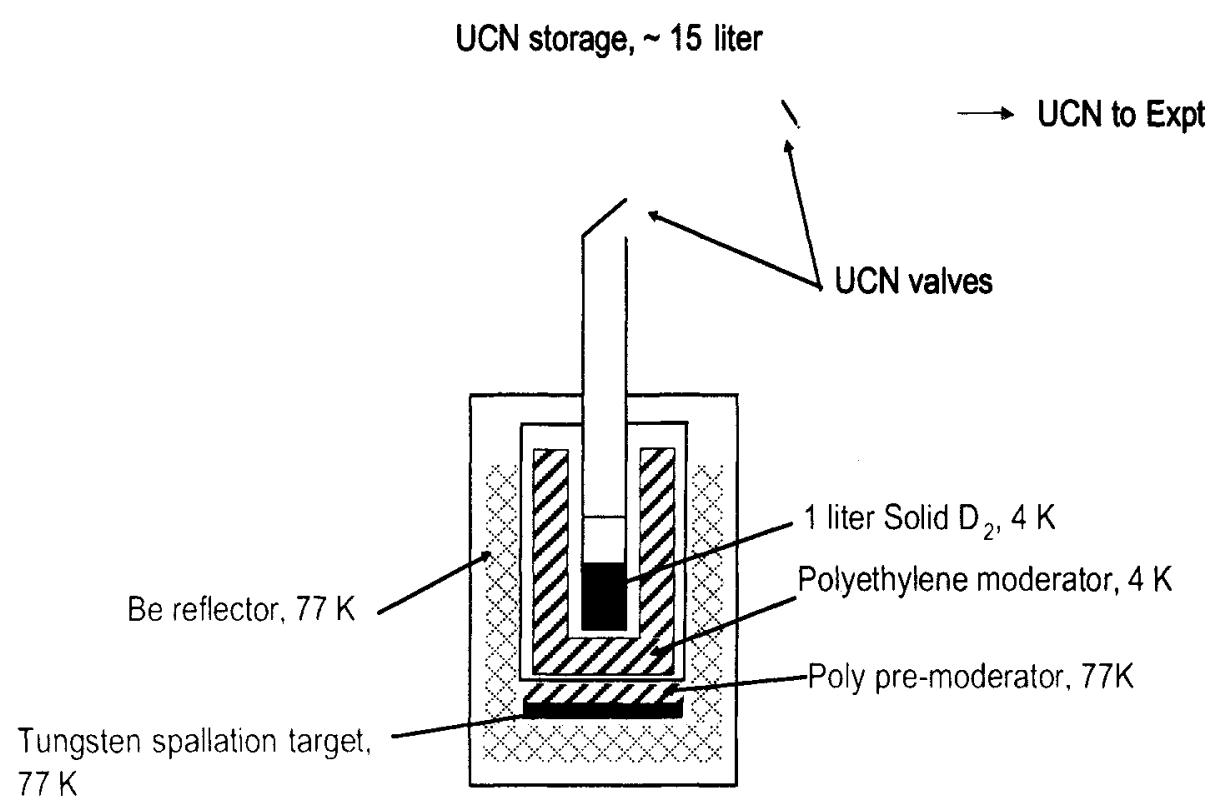


Figure 3: Dependence of UCN density in the source on the volume of solid deuterium. The line labeled $8 \mathrm{~cm}$ shows the expected result if a naïve calculation of the elastic scattering, absorption, and upscattering is correct. The data instead show an effective path length for the neutrons of $1 \mathrm{~cm}$.

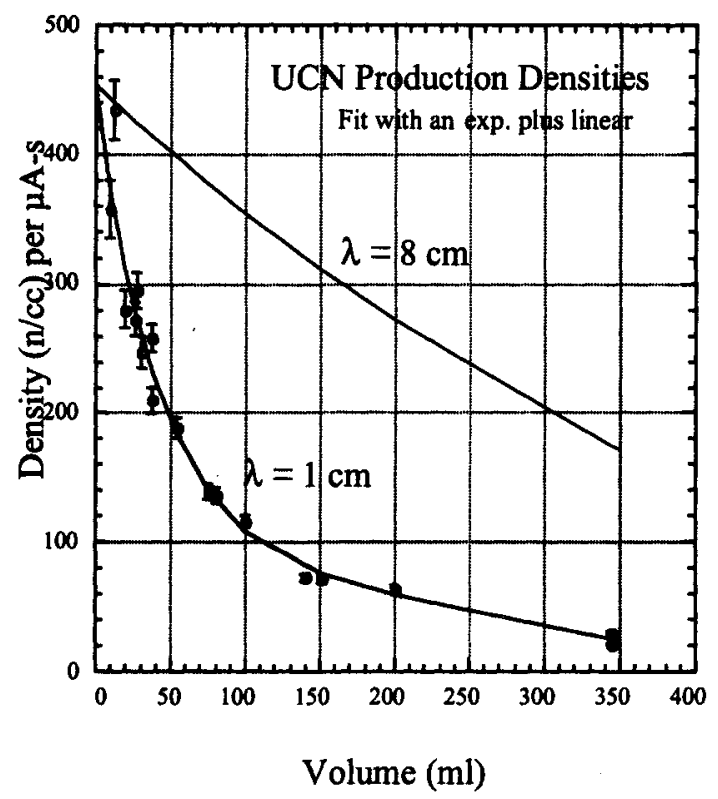

